\title{
Genetic landscape of a case of extraovarian peritoneal serous papillary carcinoma
}

\author{
ZHONGPING CHENG ${ }^{1,2}$, WEIHONG YANG ${ }^{1,2}$, JING GUO $^{1,2}$, NING LUO $^{1,2}$, LI CHEN $^{1,2}$, \\ YAN XIE $^{1,2}$, XIAOYAN QU ${ }^{1,2}$, LIPING HU $^{1,2}$, HONG DAI $^{1,2}$ and XIAOMING ZUO ${ }^{3}$ \\ ${ }^{1}$ Department of Gynecology and Obstetrics, Yangpu Hospital, School of Medicine; \\ ${ }^{2}$ Gynecology Minimally Invasive Medical Institute, School of Medicine; ${ }^{3}$ Department of Pathology, \\ Yangpu Hospital, School of Medicine, Tongji University, Shanghai 200090, P.R. China
}

Received April 8, 2015; Accepted June 21, 2016

DOI: $10.3892 / 01.2016 .4933$

\begin{abstract}
The present report aimed to study genetic alterations underlying extraovarian peritoneal serous papillary carcinoma (EPSPC), which have not previously been systematically investigated. A case of EPSPC was identified, and its genetic alterations were assessed by combining comparative genomic hybridization and whole-exome sequencing technologies to investigate the genomic landscape, including copy number variations and mutations in EPSPC. It was found that a large number of germline mutations were present, which may have predisposed the patient to the occurrence of this disease. Copy number gains were found in a range of chromosomes, including 4q, 5q, 8q, 10q, 15q, 16p, 18q, 20p, 20q and Xq. Large-scale copy number loss occurred in chromosomes $2 \mathrm{p}, 13 \mathrm{q}, 16 \mathrm{q}$, $17 \mathrm{p}$ and $17 \mathrm{q}$. Through use of whole-exome sequencing, germline mutations were widely found that were associated with cancer development, including mutations in the BRCA1, DNA repair associated (BRCA1), BRCA2, tumor protein 53, erb-b2 receptor tyrosine kinase 2, matrix metalloproteinases and ADAM metallopeptidase domain-containing genes. In addition, 165 somatic mutations, including 52 missense mutations and 7 short insertions or deletions, were also identified. In summary, the EPSPC was undergoing profound genomic rearrangement and somatic mutation, which may have led to its initiation and development, and the present study discussed the genetic basis of this highly malignant cancer.
\end{abstract}

\section{Introduction}

Serous papillary peritoneal carcinoma (SPPC) was first described in 1959 by Swerdlow (1), and this was similar to

Correspondence to: Dr Zhongping Cheng, Department of Gynecology and Obstetrics, Yangpu Hospital, School of Medicine, Tongji University, 450 Tengyue Road, Shanghai 200090, P.R. China E-mail: mdcheng18@263.net

Key words: extraovarian peritoneal serous papillary carcinoma, comparative genomic hybridization, whole-exome sequencing, mutation, copy number variation ovarian papillary cystadenocarcinoma with regard to the pathological features and to pelvic peritoneal mesothelioma when considering diffused peritoneal lesions. In 1993, extraovarian peritoneal serous papillary carcinoma (EPSPC) was formally recognized according to Gynecological Oncology Group (GOG) criteria in order to better define the SPPC patient population (2). The clinical presentations of EPSPC generally include abdominal distension and pain, ascites and increasing levels of serum cancer antigen 125 (CA125), with the absence of a pelvic mass on imaging examinations $(2,3)$. Surgical investigation and histopathological characteristics provide the evaluation for a diagnosis of EPSPC in the light of GOG criteria: Each ovary must be normally sized or enlarged by a benign process $(4.0 \mathrm{~cm}$ in largest diameter), and extraovarian site involvement must be greater than that on the ovarian surface. Furthermore, the ovarian component must be microscopically non-existent or confined to the surface epithelium of the ovary, with no evidence of cortical invasion, or must involve the ovarian epithelium and/or the underlying stroma at $<5 \times 5 \mathrm{~mm}$ in depth and extent. The tumor thus resembles serous papillary ovarian cancer (SPOC) in terms of pathological features $(2,4)$.

It is widely accepted that EPSPC is derived from the mullerian coelomic epithelium, similar to serous ovarian carcinoma (SOC) (5-7). However, histopathological and molecular evidence is accumulating to show that peritoneal carcinomas are multifocal in origin, thus differing from SOCs, which are only unifocal. Considering the histological, molecular and clinical similarities, EPSPC has traditionally been incorporated in the same staging systems of stage III/IV SPOC and managed with the similar pattern of surgical debulking and platinum-based chemotherapy as used in advanced ovarian cancer $(5,6,8)$.

EPSPC is indistinguishable from advanced ovarian cancer in terms of clinical presentation, management and molecular biology using immunohistochemistry or polymerase chain reaction (PCR)-based assays (4). However, preliminary genetic data from retrospective series have provided certain indications that may be worthy of note and research; for example, patterns of loss of heterozygosity $(\mathrm{LOH})$ at a number of chromosomal loci differ from those that are apparent in ovarian cancer. Despite effective chemotherapeutic cytoreduction and 
occasional long-term remissions (9), patients with EPSPC survive for a shorter length of time than ovarian cancer patients. Thus, any distinct molecular characteristics in EPSPC are worth investigating, and are of great importance for the illustration of unique disease characteristics. Given the lack of comprehensive molecular data, the present study performed whole-exome sequencing and comparative genomic hybridization $(\mathrm{CGH})$ analysis to investigate the molecular characteristics of a case of EPSPC, which showed distinct genetic alterations in the form of gene amplification and loss, somatic mutations and germline mutations.

\section{Materials and methods}

Case identification. A 54-year-old woman was admitted to Yangpu Hospital (Shanghai, China) in December 2012 due to a persistent low-grade fever, shortness of breath and abdominal pain. Pelvic palpation found that the patient's bilateral utero-sacral ligaments were thickened with no existence of an actual mass. The serum CA125 level was increased significantly to $2,119 \mathrm{U} / \mathrm{ml}$ (normal level, 0.00-35.00 U/ $\mathrm{ml}$ ). In addition, the serum levels of CA153 (normal range, 0.00-31.30 U/ml) and CA724 (normal range, 0.00-9.00 U/ml) were slightly increased (43.50 and $23.47 \mathrm{U} / \mathrm{ml}$, respectively), whereas the levels of CA199 (normal range, 0.00-35.00 U/ $\mathrm{ml}$ ), CA242 (normal range, 0.05-20.00 U/ml), CA50 (normal range, 0.21-25.00 U/ml), squamous cell carcinoma antigen (normal range, 0.01-2.50 ng/ml), carcinoembryonic antigen (CEA; normal concentration, $<2.50 \mathrm{ng} / \mathrm{ml}$ ) and $\alpha$-fetoprotein (AFP; normal range, $0.00-10.00 \mathrm{ng} / \mathrm{ml}$ ) were within the normal ranges. Only a small amount of ascites and pleural effusion, with no evidence of an abnormal image, were found on ultrasonography and computed tomography. In addition, gastrointestinal endoscopy failed to demonstrate any neoplasm other than chronic non-atrophic gastritis. An attendant culdocentesis was performed and the ascitic fluid was found to be positive for malignant cells consistent with metastatic adenocarcinoma. Considering the prominent increase in CA125 and the positive ascites, on December 23, 2012, laparoscopic exploration was recommended. It was observed that the bilateral ovaries $(10 \times 20 \times 30 \mathrm{~mm})$ were slightly smaller than the normal size, with some fine granular lesions on the surface. Similar diffuse micronodules were scattered on the serosal surface of the uterus, fallopian tubes, bladder, rectum, omentum, intestine and liver, as well as on the diaphragm, and the abdominal and pelvic peritoneum. Moreover, low-volume light green ascites were observed in the pelvic cavity (Fig. 1).

Post-operative histopathological evaluation of a $0.5 \times 0.5 \mathrm{~cm}$ specimen removed from the peritoneum and ovary revealed a poorly-differentiated serous adenocarcinoma, which infiltrated the ovarian surface epithelium with no stromal invasion, and involved the pelvic and abdominal peritoneum, the serosal surface of the omentum and appendix, and the pelvic lymph nodes. Invasion into the cervical vessels was of particular note (Fig. 2). Immunohistochemical staining of the lesions presented positive for CA125, cytokeratin (CK)7, Ki-67, CK19, E-cadherin, progesterone receptor (PR), EMA, estrogen receptor (ER), Wilms' tumor protein (WT1) and p53, but negative for CEA, CK5/6, AFP, CK20, human chorionic gonadotropin (HCG), calretinin, thyroid transcription factor 1 (TTF1) and villin (Fig. 3). The specimen resembled the features of SOC derived from mullerian coelomic epithelium differentiated from the peritoneal mesothelioma and metastases (3,5-7). According to the clinicopathological characteristics, the patient was eventually diagnosed with EPSPC conforming to the GOG criteria (2). A chemotherapy regimen consisting of paclitaxel $\left(135 \mathrm{mg} / \mathrm{m}^{2} / 24 \mathrm{~h}\right)$ plus cisplatin $\left(75 \mathrm{mg} / \mathrm{m}^{2}\right)$ was administered intravenously at 3 -week intervals for 6 cycles. The serum CA125 level recovered to normal, and to date, the patient has experienced disease-free survival and is under follow-up.

The research was performed according to the principles of the Declaration of Helsinki. Informed consent was obtained from the patient and the study was approved by the Ethics Committee of Yangpu Hospital.

Immunohistochemistry. Immunohistochemistry was performed using the streptavidin-biotin complex method. Briefly, tissues were fixed with $10 \%$ neutral-buffered formalin and paraffin-embedded, following by cutting into $5-\mu \mathrm{m}$ sections for immunohistochemical staining. Following the hydration and antigen repair of tissues, the sections were incubated overnight at $4^{\circ} \mathrm{C}$ with the following primary antibodies: Mouse anti-CA125 monoclonal antibody (cat. no. M-0055; Shanghai Long Island Biotec, Shanghai, China); mouse anti-CK7 monoclonal antibody (cat. no. GM7018; GeneTech, Co., Ltd., Shanghai, China); mouse anti-Ki-67 monoclonal antibody (cat. no. GT2094; GeneTech, Co., Ltd.); mouse anti-CK19 monoclonal antibody (cat. no. GT2255; GeneTech, Co., Ltd.); mouse anti-E-cadherin monoclonal antibody (cat. no. GT2153; GeneTech, Co., Ltd.); rabbit anti-PR monoclonal antibody (cat. no. F00392; Roche Diagnostics (Shanghai) Ltd., Shanghai, China); mouse anti-EMA monoclonal antibody (cat. no. GM0613; GeneTech, Co., Ltd.); rabbit anti-ER monoclonal antibody (cat. no. F02583; Roche Diagnostics (Shanghai) Ltd.); rabbit anti-WT1 polyclonal antibody (cat. no. R-0526; Shanghai Long Island Biotec); mouse anti-p53 monoclonal antibody (cat. no. R-0430; Shanghai Long Island Biotec); mouse anti-CEA monoclonal antibody (cat. no. GT2108; GeneTech, Co., Ltd.); mouse anti-CK5/6 monoclonal antibody (cat. no. GM7237; GeneTech, Co., Ltd.); rabbit anti-AFP polyclonal antibody (cat. no. GA0008; GeneTech, Co., Ltd.); mouse anti-CK20 monoclonal antibody (cat. no. GT2042; GeneTech, Co., Ltd.); mouse anti-HCG monoclonal antibody (cat. no. R-0201; Shanghai Long Island Biotec); mouse anti-calretinin monoclonal antibody (cat. no. GM3556; GeneTech, Co., Ltd.); mouse anti-TTF1 monoclonal antibody (cat. no. GM3575; GeneTech, Co., Ltd.); and rabbit anti-villin monoclonal antibody (cat. no. R-0589; Shanghai Long Island Biotec). Subsequently, the primary antibodies were detected using Dako EnVision Detection Systems Peroxidase/DAB (Rabbit/Mouse) (Dako Japan Co., Ltd., Tokyo, Japan). Negative controls were performed with normal mouse serum (Dako Japan Co., Ltd.) or phosphate-buffered saline, and sections were counterstained with hematoxylin.

Affymetrix OncoScan ${ }^{T M}$ microarray. Sample DNA was extracted using a DNeasy Blood \& Tissue kit (cat. no. 69506; Qiagen GmBH, Hilden, Germany), according to the manufacturer's instructions. A Nano Drop spectrophotometer (cat. 
no. ND-1000; Thermo Fisher Scientific Inc., Waltham, MA, USA) and $1 \%$ agarose gel electrophoresis were used to check the quantity and quality of the purified DNA. Sample DNA was digested by the NspI restriction enzyme, and adaptors were ligated to the fragment DNA to perform PCR amplification. Amplification of the DNA was performed using the OncoScan $^{\text {TM }}$ FFPE Assay kit (cat. no. 902293; Affymetrix, Santa Clara, CA, USA), according to the manufacturer's protocol.

Amplified DNA was labeled and further fragmented using an Affymetrix CytoScan HD Array Kit and Reagent Kit Bundle (cat.no. 901835; Affymetrix), according to the manufacturer's protocol. The Affymetrix OncoScan Assay User Manual (cat. no. 703038; Rev. 3; Affymetrix) was followed to obtain biotin-labeled DNA. Hybridization buffers were prepared and array hybridization was performed at $49^{\circ} \mathrm{C}$ in Hybridization Oven (cat. no. 00-0331-220V; Affymetrix). After $16 \mathrm{~h}$ of hybridization, the arrays were washed in a Fluidics Station (cat. no. 00-0079; Affymetrix) according to the Affymetrix OncoScan $^{\mathrm{TM}}$ Assay User Manual (cat. no. 703038; Rev. 3; Affymetrix). Arrays were scanned using the GeneChip ${ }^{\circledR}$ Scanner 3000 (cat. no. 00-00212; Affymetrix) and Command Console Software 3.1 (Affymetrix) with default settings. Raw data that passed quality control were further analyzed by Affymetrix ${ }^{\circledR}$ OncoScan Analysis Suite (OncoScan Console Software; Affymetrix).

Whole-exome sequencing. Primary tumor tissue consisted of blocks of fresh frozen tissue acquired at the time of laparoscopy and then frozen in liquid nitrogen. Genomic DNA was isolated from the primary tumor tissue using the AllPrep DNA/RNA Mini kit (Qiagen). Genomic DNA was isolated from blood to control for germline variants using the DNeasy Blood and Tissue kit (Qiagen). The qualified genomic DNA sample was randomly fragmented by Covaris and the size of the library fragments was mainly distributed between 150 and $200 \mathrm{bp}$. Next, adapters were ligated to each end of the resulting fragments. The adapter-ligated templates were purified by the Agencourt AMPure SPRI beads, and fragments with insert size of $\sim 176$ bp were excised. Extracted DNA was amplified by ligation-mediated PCR (LM-PCR), purified and hybridized to the SureSelect Biotiny lated RNA Library (BAITS) for enrichment. Hybridized fragments were bound to the strepavidin beads, whereas non-hybridized fragments were washed out after $24 \mathrm{~h}$. Captured LM-PCR products were subjected to a Agilent 2100 Bioanalyzer to estimate the magnitude of enrichment. Each captured library was then loaded on a Hiseq2000 platform, and high-throughput sequencing was performed for each captured library to ensure that each sample met the desired average sequencing depth. Raw image files were processed by Illumina basecalling Software 1.7 (Illumina, San Diego, CA, USA) for basecalling with default parameters, and the sequences of each individual were generated as 90/100-bp pair-end reads.

The bioinformatics analysis began from the sequencing data (raw data) generated from the Illumina pipeline. Firstly, the adapter sequence in the raw data was removed, and low quality reads that had too many Ns or a low base quality were discarded. This step produced the 'clean data'. Secondly, the Burrows-Wheeler Aligner (BWA)-MEM algorithm in the
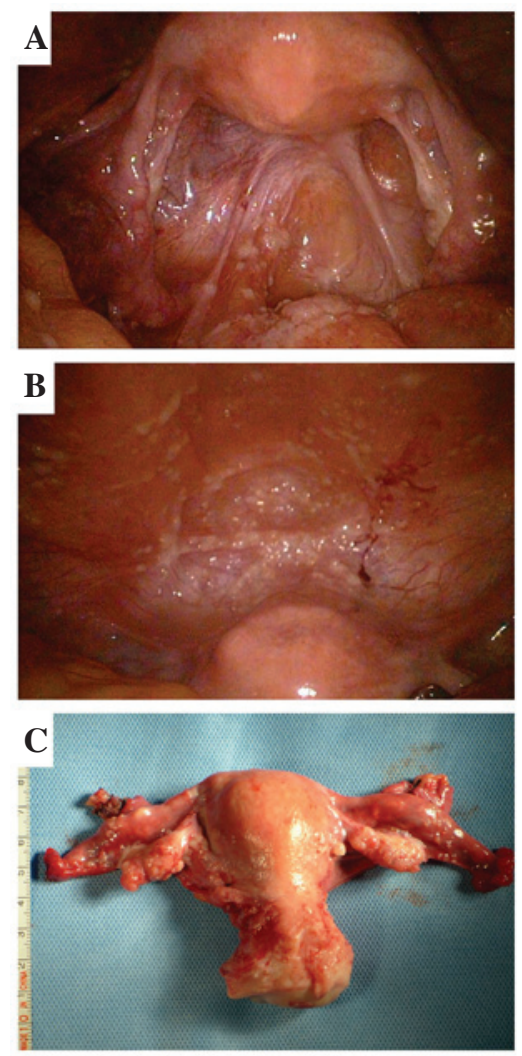

Figure 1. Exterior appearance of extraovarian peritoneal serous papillary carcinoma. (A) Metastatic nodules in the uterus, bilateral mesosalpinges, ovaries and rectal serosal surface, also in the peritoneum of the Douglas pouch and pelvic floor. (B) Metastatic nodules in the peritoneum of the uterovesical concave. (C) Samples of the uterus and ovarian fallopian tubes.

BWA software (https://sourceforge.net/projects/bio-bwa/files/) was used to perform the alignment. BWA can provide results in binary alignment/map (BAM) format files. The BAM format files were required for certain processes, such as fixing mate information of the alignment, adding read group information and removing duplicate reads caused by PCR. After these processes, the final BAM files used to do the variant calling were made ready. Single nucleotide polymorphism (SNP) analysis was performed using the Short Oligonucleotide Analysis Package for SNPs (release 1.03; http://soap.genomics. org.cn/soapsnp.html), Sequence Alignment/Map tools (release 0.1.5-22; http://www.htslib.org/download/) or Genome Analysis Toolkit (version 3.2; https://www.broadinstitute.org/ gatk/). Following this, filters were applied to obtain more confident variant results. Subsequently, AnnoDB software (Bejing Genomics Institute, Beijing, China) was used, which is in-house tool to annotate the confident variant results. The final variants were fed into the downstream advanced analysis pipeline. Quality control was present in the whole pipeline, including for the clean data, the alignment and the called variant.

Somatic single nucleotide variants (SNVs) occur in any non-germ cell of the body following conception, such as in those cells that initiate tumorigenesis. In the present study, the Variant Detection in Massively Parallel Sequencing Data platform (version 2.0; http://varscan.sourceforge.net/) was applied to identify blood sample- and tumor sample-specific SNVs by simultaneously comparing read counts, base quality and 

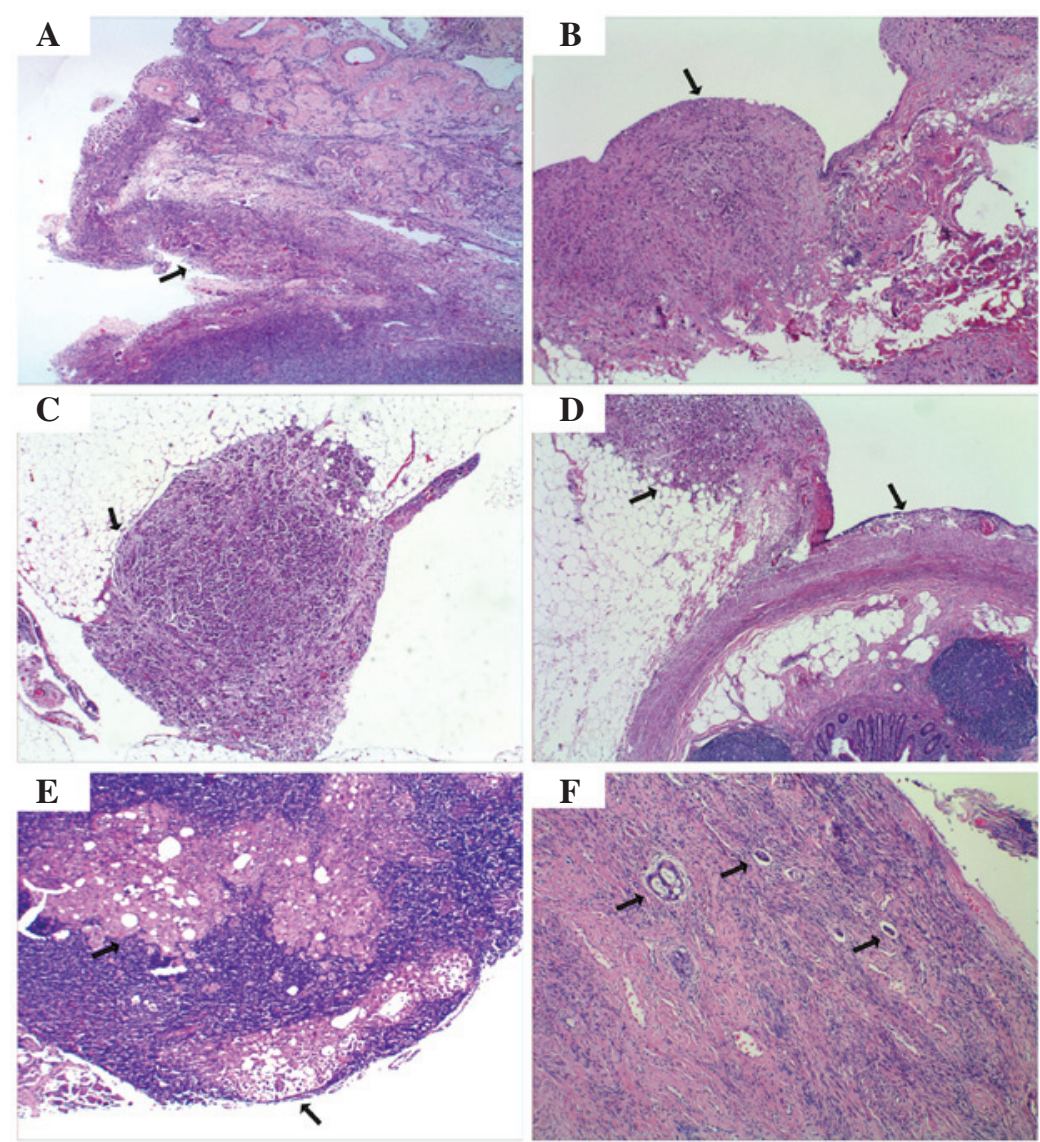

Figure 2. Histological staining of extraovarian peritoneal serous papillary carcinoma. Haematoxylin and eosin stains of the (A) ovary, (B) mesenterium, (C) omentum, (D) appendix, (E) lymph node and (F) cervix. (A-D) Cancer cells (arrows). (E) Cancer nests in the lymph node (arrows). (F) Cancer emboli in the cervical vascular tissue (arrows). (A-D) Magnification, $\mathrm{x} 40$; and (E and F) magnification, $\mathrm{x} 100$.

allele frequency between the blood and tumor tissues. Somatic InDels are mutations that occur due to small insertions or deletions of one or a few nucleotides that occur in any non-germ cell of the body after conception, such as those that initiate tumorigenesis. The strategy for InDel analysis is as follows: In the sufficient covered sites, an initial call is first made in the tumor sample, which is then compared with the normal sample to find any evidence for the event; if there is no evidence to support the InDel event in the normal sample, this site will be considered a putative somatic InDel. The probable impacts of the somatic mutations on an individual were detected using the PolyPhen-2 (http://genetics.bwh.harvard.edu/pph2/) and SIFT (http://sift.bii.a-star.edu.sg/) databases. Missense mutations predicted as 'neutral' by PolyPhen- 2 were removed. The Kyoto Encyclopedia of Genes and Genomes pathway database (http://www.genome.jp/kegg/pathway.html) was used for pathway analysis.

\section{Results}

Copy number alteration in EPSPC. The CGH microarray was applied to analyze the copy number variations by comparing tumor tissues and white blood cells (WBCs). Unexpectedly, a number of large-scale copy number alterations were identified in the tumor tissues (Fig. 4). Copy number gain occurred in a variety of chromosomes, including $4 \mathrm{q}, 5 \mathrm{q}, 8 \mathrm{q}, 10 \mathrm{q}, 15 \mathrm{q}$, 16p, 18q, 20p, 20q and Xq. In particular, chromosome 8 exhibited a high number of gene amplifications in 8q. Copy number loss occurred in numerous chromosomes, including chromosomes 1p, 1q, 2p, 6q, 8p, 9p, 11p, 12q, 13q, 16q, 17p, 17q, 19p, 19q, 21q, 22q and Xp. In particular, large-scale copy number loss was found in chromosomes $2 \mathrm{p}, 13 \mathrm{q}, 16 \mathrm{q}, 17 \mathrm{p}$ and $17 \mathrm{q}$.

Gene mutation in EPSPC. Whole-exome sequencing was applied to investigate gene mutational spectra and insertion or deletion. A total of 14.6 billion bases of sequence were obtained (clean data/raw data, 92.13\%) in tumor tissue, and a total of 15.2 billion bases of sequence were obtained (clean data/raw data, 92.31\%) in WBC DNA (germline DNA). A total effective yield of 12,431 and 12,812 Mb bases was found for tumor tissue DNA and WBC DNA, respectively. The average read length was $89 \mathrm{bp}$ for each. A total of 81,339 mutations were identified in the germline DNA, including 8,062 missense mutations and 9,563 synonymous mutations (data not shown). A total of 11,286 short insertions or deletions were identified in the germline DNA, including 187 frameshift insertions/deletions, 62 non-frameshift insertions and 77 non-frameshift deletions (data not shown).

WBC DNA was analyzed to identify germline DNA mutation (data not shown). The results showed that a few important genes that are associated with cancer development were mutated, such as BRCA1, DNA repair associated (BRCA1), which has 4 missense mutation sites, including rs1799966 


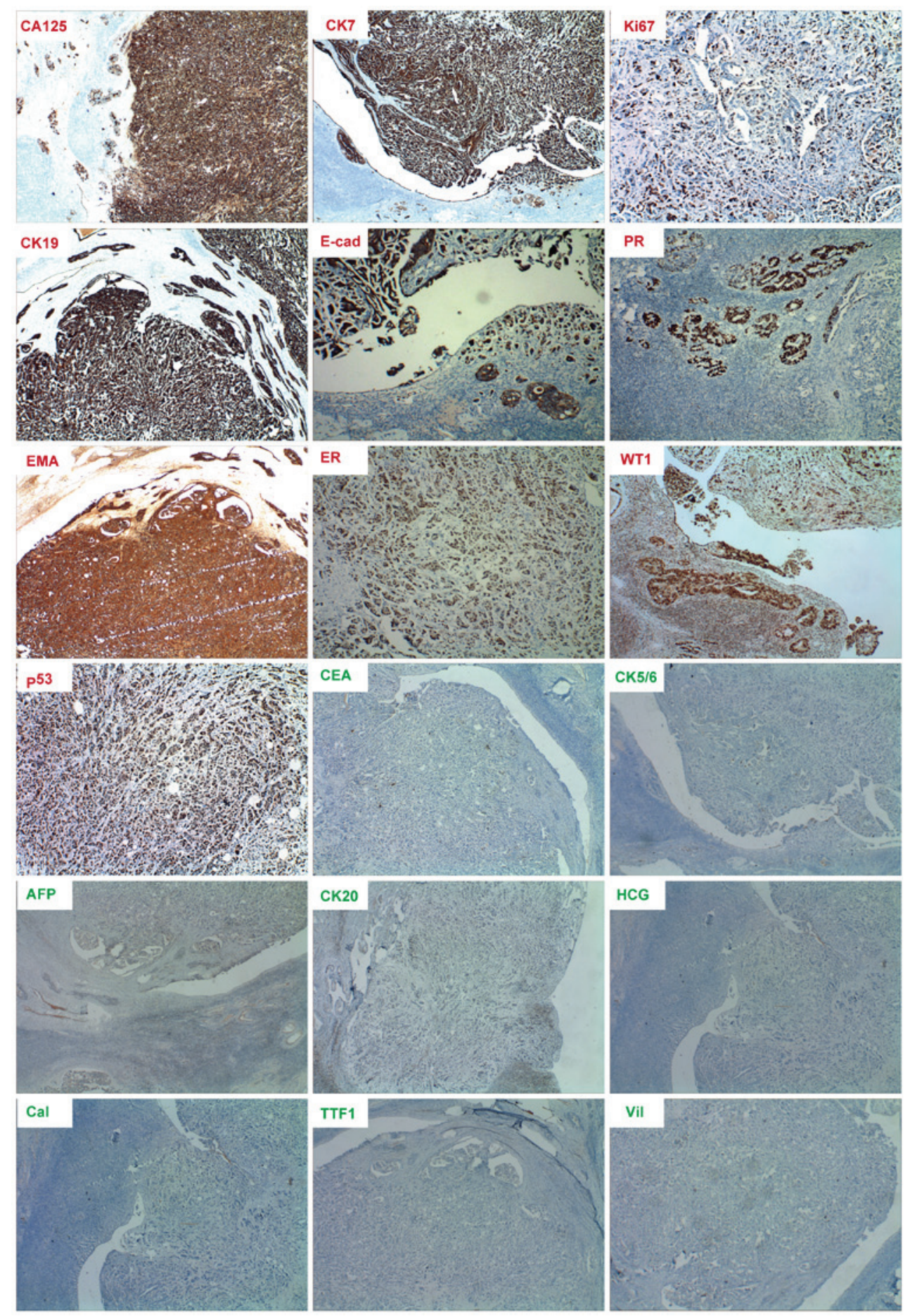

Figure 3. Immumohistochemical staining of cancer biomarkers in extraovarian peritoneal serous papillary carcinoma. Positive staining (red lables) for cancer antigen 125 (CA125), cytokeratin 7 (CK7), CK19, E-cadherin (E-cad), epithelial membrance antigen (EMA), estrogen receptor (ER), progesterone receptor (PR), Ki-67, tumor protein 53 (p53) and Wilms' tumor protein (WT1). The results show negative staining for $\alpha$-fetoprotein (AFP), calretinin (Cal), carcinoembryonic antigen (CEA), CK5/6, CK20, human chorionic gonadotropin (HCG), thyroid transcription factor 1 (TTF1) and villin. Magnification for ER, Ki-67 and $\mathrm{p} 53$ is $\mathrm{x} 100$, while magnification for the remainder is $\mathrm{x} 40$.

(Agt/Ggt), rs16942 (aAa/aGa), rs16941 (gAa/gGa) and rs799917 (cCg/cTg). BRCA2 has 1 missense mutation (rs169547; $\mathrm{gTa} / \mathrm{gCa}$ ). Tumor protein 53 (TP53) has 1 missense mutation (rs1042522, cCc/cGc). Erb-b2 receptor tyrosine kinase 2 (ERBB2) has 1 missense mutation (rs1058808, Ccc/Gcc). Additionally, oncogenes such as epidermal growth factor receptor (EGFR) and KRAS proto-oncogene, GTPase (KRAS), and the tumor suppressor gene, WNT signaling pathway regulator, also exhibited mutations. Moreover, a number of cell migration-associated genes were mutated, such as matrix metallopeptidase (MMPs) and ADAM metallopeptidase domain-containing gene (ADAMs) family members (Fig. 2).

Next, 165 somatic mutations were identified by comparing tumor tissue DNA with WBC DNA, and the majority were novel mutations (152/165), including 52 missense mutations, 15 synonymous mutations, 1 stop-gain 


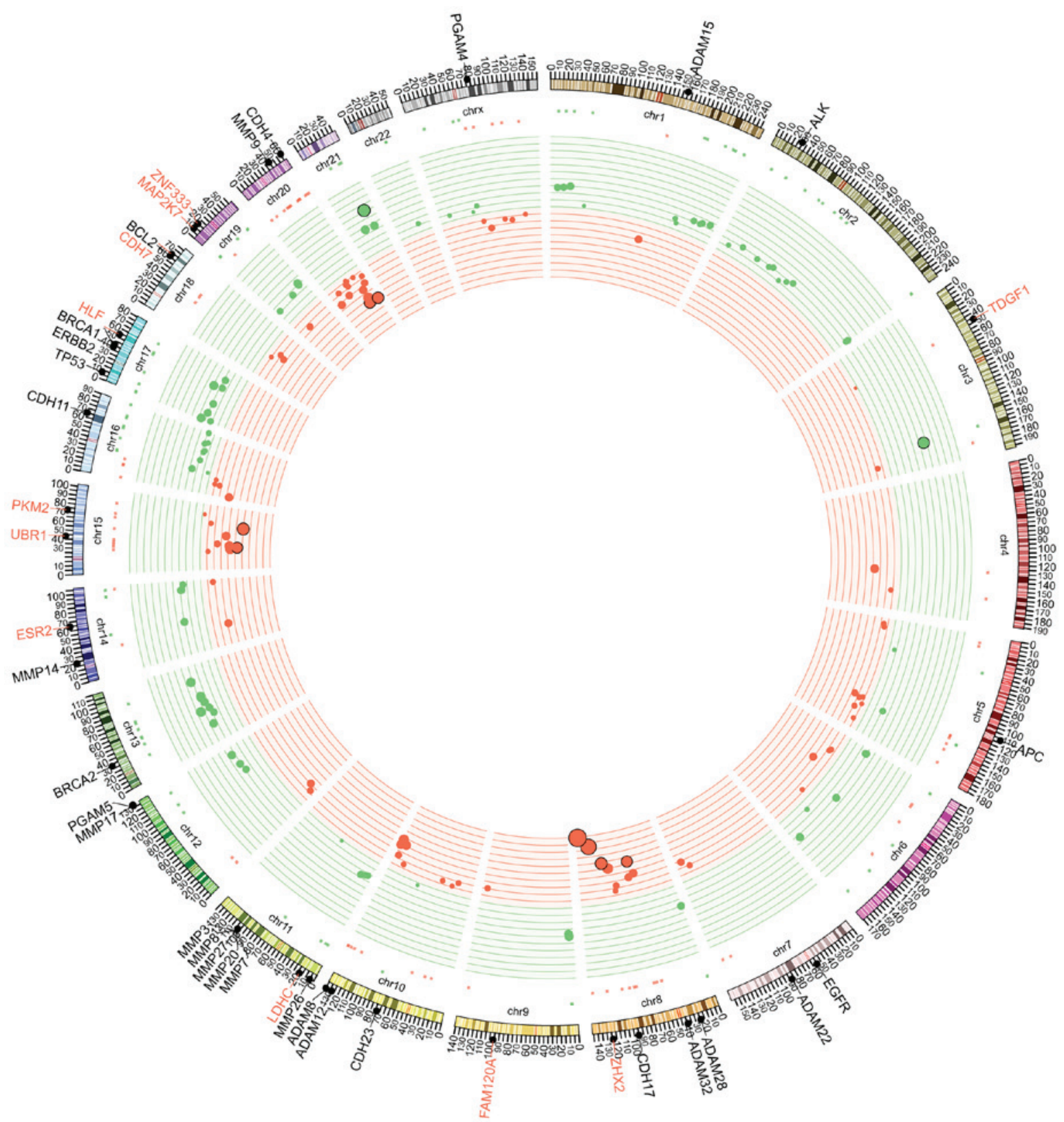

Figure 4. Genomic landscape of extraovarian peritoneal serous papillary carcinoma. Circles from outside to inside depict the following: i) Chromosomes and validated mutations revealed by whole exome sequencing; gene names in red indicate somatic mutations, while gene names in black indicate germline mutations; ii) Copy number as a function of genomic coordinates; red represents amplifications and green represents deletions.

mutation (immunoglobulin-like and fibronectin type III domain containing 1), 7 short insertions/deletions and 3 novel mutations, among others (data not shown). In the functional prediction of these mutations, 70 potential driver mutations were screened out (data not shown), some of which may be important for cancer metastasis and growth, such as cadherin 7 (CDH7), family with sequence similarity 120A (FAM120A) and ER 2 (ESR2). However, the majority of these mutations were novel findings, which are unknown to cancer metastasis and require further functional characterization.

\section{Discussion}

The present study investigated the copy number variation and mutational spectra of a case with EPSPC, which may provide novel insights into the course of disease evolution. Copy number loss was previously reported in a study by Cass et al (10), which screened for LOH at 39 chromosomal loci in 26 EPSPC and 37 SOC cases. Loci with LOH were found in $>30 \%$ of EPSPC cases in chromosomes 12p, 17p, 17q and 18q, as compared with large-scale loci with LOH in $>30 \%$ of SOC cases, located in chromosomes 4q, 5q, 6p, 6q, 9p, 9q, 12p, 12q, 13q, 15q, 16q, $17 \mathrm{p}, 17 \mathrm{q}, 18 \mathrm{q}, 19 \mathrm{p}, 19 \mathrm{q}, 22 \mathrm{q}$ and Xq. It appeared that genomic stability was less frequent in EPSPC compared with SOC. Huang et al similarly profiled 52 EPSPC and 33 SOC cases for LOH at 22 microsatellite markers (11). LOH was commonly observed in the EPSPC cases in loci 6q, 9p, 17p, 17q and Xq, while SOC cases commonly harbored LOH in 1p, 7q, 11p, 17p and $17 q$. In the present study, the patient showed a wide range of large-scale copy number alterations across chromosomes. 


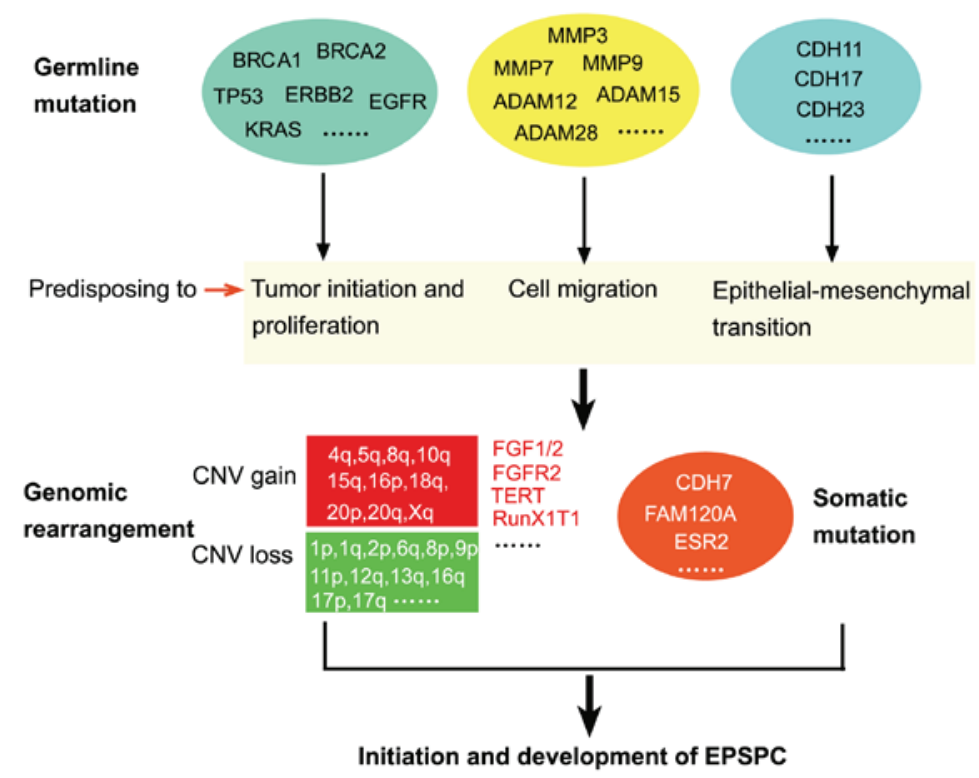

Figure 5. Ideogram illustration of genomic alterations that may be involved in the initiation and development of EPSPC. Germline mutations may predispose an individual to the disease, and then undergoing genomic rearrangement and somatic mutation may lead to the occurrence and development of EPSPC. BRCA1, BRCA1, DNA repair associated; TP53, tumor protein 53; ERBB2, erb-b2 receptor tyrosine kinase 2; EGFR, epidermal growth factor receptor; KRAS, KRAS proto-oncogene, GTPase; ADAM, ADAM metallopeptidase domain-containing; MMP, matrix metalloproteinase; CDH, cadherin; FGF, fibroblast growth factor; FGFR, FGF receptor; TERT, telomerase reverse transcriptase; RUNX1T1, RUNX1 translocation partner 1; FAM120A, family with sequence similarity 120A; ESR2, estrogen receptor 2; CNV, copy number variation; EPSPC, extraovarian peritoneal serous papillary carcinoma.

In accordance with the previous study by Huang et al (11), this patient had copy number loss in 6q, 9p, 17p and 17q. However, the patient also exhibited many more copy number losses than previous studies, with distinct copy number loss in 1q, 2p, 2q and Xp when compared with SOC. Additionally, numerous copy number gains were found across the chromosomes, including $4 \mathrm{q}$, 5q, 8q, 10q, 15q, 16p, 18q, 20p, 20q and Xq, implying a profound genomic rearrangement in the evolution of EPSPC. Furthermore, the present study analyzed the genes located in these gain regions and found certain notable indications, such as in fibroblast growth factor (FGF)2 (located in 4q), FGF1 (located in 5q) and FGF receptor (FGFR)2 (located in 10q), where the amplification of these genes suggested that FGF-FGFR2 signaling may be involved in tumor progression. The study also found other cancer-related genes located in copy number gain regions, such as telomerase reverse transcriptase in 5q, runt-related transcription factor 1 in 8q, encoding mitogen-activated protein kinase kinase 5 in $15 q$ and mitogen-activated protein kinase 3 in 16p. Collectively, these copy number losses or gains may play essential roles in the development of EPSPC.

There were numerous germline missense mutations in the present case, suggesting that the cancer susceptibility genes, including BRCA1, BRCA2, TP53, ERBB2, EGFR and KRAS, predisposed the patient to the occurrence of the cancer. BRCA1 and BRCA2 had been reported to be frequently mutated in EPSPC (12), and BRCA1 mutation may be associated with a multifocal pathogenesis in such patients (13). BRCA1, BRCA2, TP53, ERBB2, EGFR and KRAS also exhibit mutations in SOC (14-16), and thus cannot be used as a unique feature to differentiate it from EPSPC. However, unexpectedly, numerous MMP and ADAM family members exhibited mutations in the present patient, which may have been a predisposing factor for the high mobility of the cancer cells and caused the wide distribution of cancer cells in the peritoneum.
Furthermore, 165 somatic mutations were found in the present case; the majority of them were novel mutations, quite different with the well-known driver oncogenes. For the functional prediction of these mutations, 70 potential driver genes were shown, some of which may be associated with tumor development. $\mathrm{CDH} 7$, which is critical for epithelial-mesenchymal transition and cell migration (17), may have an impact on the metastasis of tumor cells. FAM120A may act in the transport of mRNA in the cytoplasm, and is a vital component of oxidative stress-induced survival signaling. FAM120A could act as activator of src family kinases to phosphorylate and activate phosphoinositide 3-kinase (18). ESR2 encodes ER $\beta$, which binds estrogens with an affinity similar to that of ER $\alpha$, and activates the expression of reporter genes containing estrogen response elements in an estrogen-dependent manner, as an ER to promote tumor growth (19). Collectively, profound genomic rearrangements and somatic mutations may lead to the initiation and development of EPSPC (Fig. 5). However, the majority of these somatic mutations are novel and require further functional characterization.

In conclusion, the present study identified large-scale copy number variations and the mutational spectra of EPSPC. Germline DNA mutations appear to be much more profound than somatic mutations, as numerous mutations were associated with cell proliferation and migration, which suggested that the germline mutations of this patient may predispose them to the occurrence of EPSPC. Further studies are required to identify the functional significance of these complex copy number variations and gene mutations.

\section{References}

1. Swerdlow M: Mesothelioma of the pelvic peritoneum resembling papillary cystadenocarcinoma of the ovary; case report. Am J Obstet Gynecol 77: 197-200, 1959. 
2. Bloss JD, Liao SY,BullerRE,Manetta A,Berman ML,McMeekin S, Bloss LP and DiSaia PJ: Extraovarian peritoneal serous papillary carcinoma: A case-control retrospective comparison to papillary adenocarcinoma of the ovary. Gynecol Oncol 50: 347-351, 1993.

3. Bhuyan P, Mahapatra S, Sethy S, Parida P and Satpathy S: Extraovarian primary peritoneal papillary serous carcinoma. Arch Gynecol Obstet 281: 561-564, 2010.

4. Pentheroudakis G and Pavlidis N: Serous papillary peritoneal carcinoma: Unknown primary tumour, ovarian cancer counterpart or a distinct entity? A systematic review. Crit Rev Oncol Hematol 75: 27-42, 2010.

5. Goodman MT and Shvetsov YB: Rapidly increasing incidence of papillary serous carcinoma of the peritoneum in the United States: Fact or artifact? Int J Cancer 124: 2231-2235, 2009.

6. Dubeau L: The cell of origin of ovarian epithelial tumours. Lancet Oncol 9: 1191-1197, 2008.

7. Grant DJ, Moorman PG, Akushevich L, Palmieri RT, Bentley RC and Schildkraut JM: Primary peritoneal and ovarian cancers: An epidemiological comparative analysis. Cancer Causes Control 21: 991-998, 2010

8. Gao YN, Liu JX, Wang W, Li WF and Tang WS: Comparison of primary extraovarian peritoneal serous papillary carcinoma with stage III-IV ovarian papillary serous carcinoma. Zhonghua Zhong Liu Za Zhi 27: 171-173, 2005 (In Chinese).

9. Kowalski LD, Kanbour AI, Price FV, Finkelstein SD, Christopherson WA, Seski JC, Naus GJ, Burnham JA, Kanbour-Shakir A and Edwards RP: A case-matched molecular comparison of extraovarian versus primary ovarian adenocarcinoma. Cancer 79: 1587-1594, 1997.

10. Cass I, Baldwin RL, Fasylova E, Fields AL, Klinger HP, Runowicz CD and Karlan BY: Allelotype of papillary serous peritoneal carcinomas. Gynecol Oncol 82: 69-76, 2001.

11. Huang LW, Garrett AP, Schorge JO, Muto MG, Bell DA, Welch WR, Berkowitz RS and Mok SC: Distinct allelic loss patterns in papillary serous carcinoma of the peritoneum. Am J Clin Pathol 114: 93-99, 2000.
12. Bandera CA, Muto MG, Schorge JO, Berkowitz RS, Rubin SC and Mok SC: BRCA1 gene mutations in women with papillary serous carcinoma of the peritoneum. Obstet Gynecol 92: 596-600, 1998

13. Schorge JO, Muto MG, Welch WR, Bandera CA, Rubin SC, Bell DA, Berkowitz RS and Mok SC: Molecular evidence for multifocal papillary serous carcinoma of the peritoneum in patients with germline BRCA1 mutations. J Natl Cancer Inst 90: 841-845, 1998.

14. McBride DJ, Etemadmoghadam D, Cooke SL, Alsop K, George J, Butler A, Cho J, Galappaththige D, Greenman C, Howarth KD, et al: Tandem duplication of chromosomal segments is common in ovarian and breast cancer genomes. J Pathol 227: 446-455, 2012.

15. Castellarin M, Milne K, Zeng T, Tse K, Mayo M, Zhao Y, Webb JR, Watson PH, Nelson BH and Holt RA: Clonal evolution of high-grade serous ovarian carcinoma from primary to recurrent disease. J Pathol 229: 515-524, 2013.

16. Bashashati A, Ha G, Tone A, Ding J, Prentice LM, Roth A, Rosner J, Shumansky K, Kalloger S, Senz J, et al: Distinct evolutionary trajectories of primary high-grade serous ovarian cancers revealed through spatial mutational profiling. J Pathol 231: 21-34, 2013.

17. Winklmeier A, Contreras-Shannon V, Arndt S, Melle C and Bosserhoff AK: Cadherin-7 interacts with melanoma inhibitory activity protein and negatively modulates melanoma cell migration. Cancer Sci 100: 261-268, 2009.

18. Tanaka M, Sasaki K, Kamata R, Hoshino Y, Yanagihara K and Sakai R: A novel RNA-binding protein, Ossa/C9orf10, regulates activity of Src kinases to protect cells from oxidative stress-induced apoptosis. Mol Cell Biol 29: 402-413, 2009.

19. Leung YK, Mak P, Hassan S and Ho SM: Estrogen receptor (ER)-beta isoforms: A key to understanding ER-beta signaling. Proc Natl Acad Sci USA 103: 13162-13167, 2006. 